\title{
Erratum to: Paramagnetic labelling of proteins and oligonucleotides for NMR
}

\author{
Xun-Cheng Su $\cdot$ Gottfried Otting
}

Published online: 19 February 2011

(C) Springer Science+Business Media B.V. 2011

Erratum to: J Biomol NMR (2010) 46:101-112

DOI 10.1007/s10858-009-9331-1

Table 3 in this review contains some misquoted results. Please find the corrected Table below.

(1) The $\mathrm{OH}$ group in tags $\mathrm{T} 2(\mathrm{R}, \mathrm{R})$ and $\mathrm{T} 2(\mathrm{~S}, \mathrm{R})$ should be a $\mathrm{COOH}$ group in Fig. 5. Please find the corrected figure below.
The reference to Vlasie MD et al. should have read: Vlasie MD, Comuzzi C, van den Nieuwendijk AM, Prudêncio M, Overhand M, Ubbink M (2007) Long-rangedistance NMR effects in a protein labeled with a lanthanide-DOTA chelate. Chem Eur J13:1715-1723

The online version of the original article can be found under doi:10.1007/s10858-009-9331-1.

X.-C. Su · G. Otting $(\bowtie)$

Research School of Chemistry, The Australian National

University, Canberra, ACT 0200, Australia

e-mail: gottfried.otting@anu.edu.au 
Table 3 Maximal residual dipolar couplings of protein backbone amides, ${ }^{1} D_{\mathrm{NH}}$, obtained with different lanthanide binding tags

\begin{tabular}{|c|c|c|c|c|c|}
\hline Lanthanide binding tag & Protein & Metal ion & Magnetic Field/Tesla & Maximal $^{\mathrm{a}}{ }^{1} D_{\mathrm{NH}} / \mathrm{Hz}$ & Reference \\
\hline $\mathrm{sLBT}^{\mathrm{b}}$ & Ubiquitin & $\mathrm{Tm}$ & 18.8 & About 5 & Martin et al. (2007) \\
\hline $\mathrm{dLBT}^{\mathrm{c}}$ & Ubiquitin & $\mathrm{Tm}$ & 18.8 & About -17 & Martin et al. (2007) \\
\hline $\mathrm{LBT}^{\mathrm{d}}$ & Galectin-3 & Dy & 21.1 & \pm 15 & Zhuang et al. (2008) \\
\hline $\mathrm{LBT}^{\mathrm{e}}$ & Arginine repressor & $\mathrm{Tm}$ & 18.8 & 22.6 & Su et al. (2008a) \\
\hline $\mathrm{T} 1^{\mathrm{f}}$ & $\mathrm{F}_{1} \mathrm{~F}_{\mathrm{o}}$ ATP synthase & $\mathrm{Tm}$ & 18.8 & -10.6 & Kamen et al. (2007) \\
\hline $\mathrm{T} 1^{\mathrm{f}}$ & $\mathrm{F}_{1} \mathrm{~F}_{\mathrm{o}}$ ATP synthase & $\mathrm{Tb}$ & 21.1 & -8.1 & Kamen et al. (2007) \\
\hline$T 2^{f}$ & Trigger factor & Dy & 18.8 & 10.5 & Ikegami et al. (2004) \\
\hline$T 3^{\mathrm{f}}$ & Trigger factor & Dy & Not reported & About 9 & Haberz et al. (2006) \\
\hline $\mathrm{T} 7^{\mathrm{f}}$ & Pseudoazurin & $\mathrm{Tm}$ & 14.1 & 26 & Keizers et al. (2008) \\
\hline$T 8^{f}$ & Arginine repressor & $\mathrm{Tm}$ & 18.8 & 12.7 & Su et al. (2008b) \\
\hline $\mathrm{CaM}(\mathrm{Tb})_{4}{ }^{\mathrm{g}}$ & DHFR-M13 & $\mathrm{Tb}$ & 14.1 & -7.4 & Feeney et al. (2001) \\
\hline
\end{tabular}

a The largest numerical value is reported

b The peptide GPGYIDTNNDGWYEGDELLA fused to the N-terminus

c The peptide GPGYIDTNNDGWIEGDELYIDTNNDGWIEGDELLA fused to the N-terminus

d The peptide YIDTNNDGWYEGDELLA fused to the C-terminus

e Tag shown in Fig. 2

${ }^{f}$ Tags T1 to T8 are depicted in Fig. 5

g Calmodulin loaded with four $\mathrm{Tb}^{3+}$ ions

Fig. 5 Lanthanide binding tags for site-specific labelling of proteins with metal ions. The tags $\mathrm{T} 1$ and $\mathrm{T} 8$ are commercially available<smiles>CS(=O)(=O)SCCNC(=O)CN(CCN(CC(=O)O)CC(=O)O)CCN(CC(=O)O)CC(=O)O</smiles>

T1

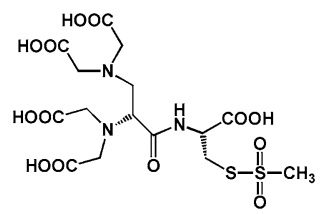

$\mathrm{T} 2(R, R)$

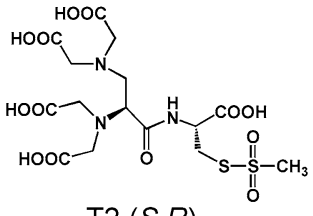

$\mathrm{T} 2(S, R)$

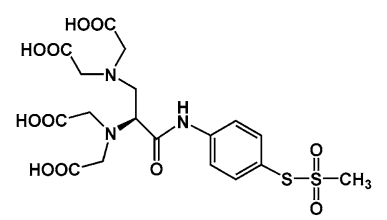

T3 $(R)$<smiles>CS(=O)(=O)Sc1ccc(NC(=O)C(CN(CC(=O)O)CC(=O)O)N(CC(=O)O)CC(=O)O)cc1</smiles>

T3 (S)<smiles>CS(=O)(=O)OCCNC(=O)CN(CCN(CC(=O)O)CC(=O)O)CCN(CC(=O)O)CC(=O)O</smiles>

T4

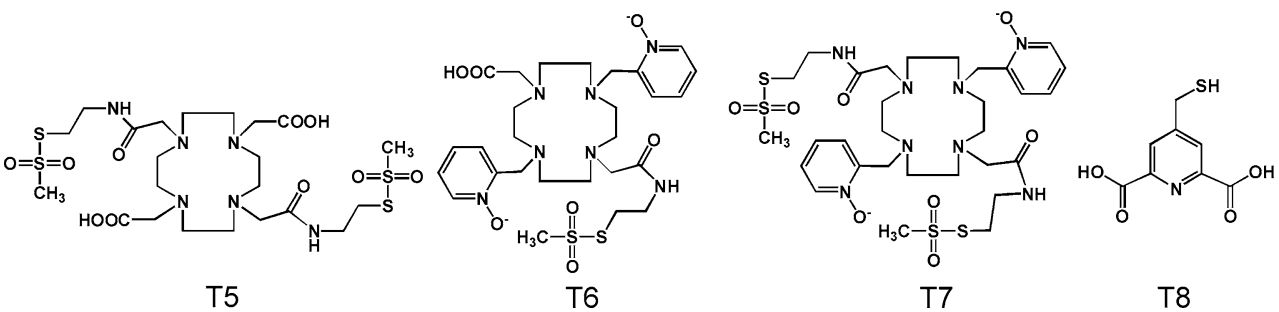

